\title{
Distance-aware Coordinated Multi-Point Transmission for Terahertz Band Communication
}

\author{
Alexis A. Dowhuszko ${ }^{1}$, Víctor P. Gil Jiménez ${ }^{2}$, Borja Genovés Gumán ${ }^{2}$, and Ana I. Pérez-Neira ${ }^{1,3}$ \\ ${ }^{1}$ Centre Tecnològic de les Telecomunicacions de Catalunya (CTTC/CERCA), Barcelona, Spain \\ ${ }^{2}$ Signal Theory and Communications Department, Universidad Carlos III de Madrid (UC3M), Madrid, Spain \\ ${ }^{3}$ Department of Signal Theory and Communications, Universitat Politecnica de Catalunya (UPC), Barcelona, Spain \\ Email: alexis.dowhuszko@cttc.es; vgil@tsc.uc3m.es; bgenoves@tsc.uc3m.es; ana.perez@cttc.es
}

\begin{abstract}
Terahertz (THz) band communication has abundant spectral resources to accommodate multiple broadband carriers. However, the strong path loss attenuation and the limited transmission power affect the maximum number of carriers that can be activated. A simple way to address this limitation consists in transmitting the same message from multiple transmission points, using Joint-Transmission (JT) Coordinated Multi-Point (CoMP). However, the distance-frequency-dependent molecular absorption that THz channels experience makes the implementation of JTCoMP challenging. In this paper, we propose a decentralized scheme that takes into account the effect of distance to select the per-carrier transmit beamforming weights and powers. For this purpose, low-rate feedback information is reported from the receiver to the transmission points, such that decisions performed locally take into account the ones made in the coordinated set. Notable sum data rate gains are observed when compared to the baseline schemes, where transmission power is equally divided among carriers or independently allocated using waterfilling. As expected, the accuracy of the reported channel phase information, the number active transmission points, and the distance-dependent frequency selectivity of the THz channel affect the sum data rate that multi-carrier JT-CoMP can provide.
\end{abstract}

\section{INTRODUCTION}

Terahertz (THz) band communication $(0.1-10 \mathrm{THz})$ has potential to alleviate the spectrum scarcity and capacity limitations of contemporary wireless systems [1]. Though $\mathrm{THz}$ band offers a very broad bandwidth, the transmission distance is typically short due to very high path loss attenuation [2]. This is because, apart from high spreading loss, there is also strong molecular absorption that depends on the mixture of gases and water vapor in the atmosphere. This effect results in a frequency-selective path loss, whose variability grows with distance [3]. Traditionally, most of contemporary research on $\mathrm{THz}$ communication has focused on characterizing the supported data rate in point-to-point links, where the usable bandwidth reduces notably for distances beyond few meters [2]. However, such an approach limits the potential of $\mathrm{THz}$ technology, as longer transmission distances are feasible if multiple transmission points cooperate to serve a receiver.

Coordinated Multi-Point (CoMP) transmission is a technology that enables the cooperation of multiple transmission points to serve a common user [4]. In Joint Transmission (JT) CoMP, the same data stream is simultaneously transmitted from multiple points, increasing the sum power and providing coherent combining gain when some sort of Channel State Information (CSI) is available in transmission. For single-carrier
JT-CoMP, it was shown in [5] that sparsely quantized channel phase information and long-term power information is enough to provide a received SNR gain close to the one with full CSI. However, for a JT-CoMP system with multiple carriers, the achievable data rate after carrier aggregation has not been characterized so far, particularly when there is limited CSI in transmission and a per-transmission point power constraint.

Joint Power Allocation (JPA) algorithms can bring further improvements in the sum data rate of JT-CoMP over frequency-selective carriers. For example, the authors of [6], [7] characterized the sum data rate of JT-CoMP when multiple non-coherent transmission points (no CSI) serve a common user over frequency-selective channels. However, when limited feedback information is available (partial CSI) [8], simple Quantized Co-Phasing (QCP) scheme can be used to transmit information coherently on a per-carrier basis [9], [10] and improve the sum data rate of JT-CoMP even further. Though the combination of JPA and QCP looks natural, it has not been addressed before in the context of multi-carrier JT-CoMP systems. This characterization is critical, since both technologies will be needed to enhance the coverage of $\mathrm{THz}$ technology and make it relevant for future ultra-dense small cell deployments.

In this paper we study the sum data rate that JPA and QCP can provide in a JT-CoMP system with multiple THz carriers. Note that this problem is similar to the one presented in [11] but, due to transmit antennas have different geographical locations, power constraint is set per transmission point. In particular, we start by presenting the optimization problem from a centralized perspective and, after that, we provide an equivalent cooperative decentralized approach that solves the same problem exchanging low-rate feedback information. Notable performance gains are observed when multiple points coordinate their transmission jointly, taking into account the effect that distance has on the frequency-selective attenuation that $\mathrm{THz}$ carriers experience. Even though the performance of different distributed antenna systems (also called distributed MIMO) has been extensively studied in the literature in references like [12], the key difference in this paper is the absence of a powerful unit that relies on global CSI to perform all sophisticated signal processing in a centralized location.

The rest of the paper is organized as follows: Section II presents the system model and the centralized optimization problem to be solved. Section III discusses briefly the division of the centralized problem into two parts: QCP and JPA. 


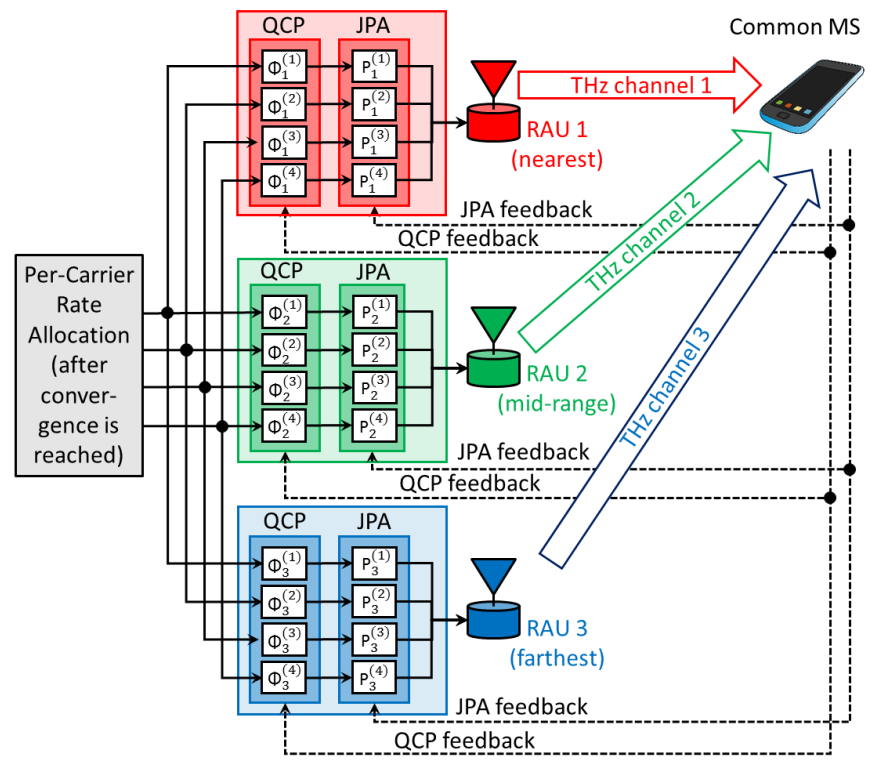

Fig. 1: JT-CoMP scenario composed by $K=4$ carriers, $M=3$ power-constrained RAUs, and a MS. Dashed lines provide the feedback information that each RAU $m$ needs to find the co-phasing weights $\phi_{m}^{(k)}$ and transmission power $p_{m}^{(k)}$ on each carrier $k$. Thick arrows represent distance-frequency-dependent $\mathrm{THz}$ channels. Rate allocation on carrier domain is done after QCP and JPA convergence.

Section IV derives a cooperative decentralized algorithm to implement the JPA part using low-rate feedback information, whereas Section $\mathrm{V}$ analyzes the corresponding sum data rate performance. Finally, conclusions are drawn in Section VI.

Notation: Matrices (vectors) are indicated with bold capital (lower case) letters. Complex and real scalar numbers are represented using normal lower case letters. Superscripts $(\cdot)^{*}$, $(\cdot)^{\mathrm{T}}$, and $(\cdot)^{\mathrm{H}}$ represent the conjugate, transpose, and Hermitian of a vector or matrix, respectively. Finally, $\operatorname{diag}\{\mathbf{d}\}$ is a diagonal matrix whose main diagonal elements are in vector $\mathbf{d}$.

\section{SYSTEM MODEL}

Consider the JT-CoMP scenario presented in Fig. 1, where $M$ Remote Antenna Units (RAUs) serve a Mobile Station (MS) on $K$ equal-size carriers located on different portions of the $\mathrm{THz}$ band. When the same information symbols are transmitted from each RAU (i.e., one data stream per carrier), the signal that MS receives on carrier $k$ is

$$
y^{(k)}=\mathbf{h}^{(k) \mathrm{T}} \mathbf{x}^{(k)}+n^{(k)} \quad k=1, \ldots, K,
$$

where $\mathbf{h}^{(k)} \in \mathbb{C}^{M \times 1}$ is the aggregate channel gain vector from the coordinated set of RAUs to the MS, $\mathbf{x}^{(k)} \in \mathbb{C}^{M \times 1}$ is the distributed transmit signal vector (after co-phasing and power allocation are applied to the information symbols), and $n^{(k)}$ is additive white Gaussian noise with power $P_{\mathrm{N}}$. Therefore, the sum data rate of JT-CoMP after carrier aggregation becomes

$$
R^{\mathrm{JT}}=W \sum_{k=1}^{K} \log _{2}\left(1+\bar{\gamma}^{(k)}\right)
$$

where $W$ is the communication bandwidth per carrier,

$$
\bar{\gamma}^{(k)}=\frac{\mathbb{E}\left\{\left|\mathbf{h}^{(k) \mathrm{T}} \mathbf{x}^{(k)}\right|^{2}\right\}}{\mathbb{E}\left\{\left|n_{k}\right|^{2}\right\}}=\frac{\mathbf{h}^{(k) \mathrm{T}} \mathbf{Q}^{(k)} \mathbf{h}^{(k) *}}{P_{\mathrm{N}}}
$$

is the mean received SNR on carrier $k$, and $\mathbf{Q}^{(k)}$ is the signal covariance matrix. The optimization of signal covariance matrix can be divided into two parts, i.e.,

$$
\mathbf{Q}^{(k)}=\operatorname{diag}\left\{\sqrt{\mathbf{p}^{(k)}}\right\}\left(\mathbf{u}^{(k)} \mathbf{u}^{(k) \mathrm{H}}\right) \operatorname{diag}\left\{\sqrt{\mathbf{p}^{(k)}}\right\},
$$

where $\mathbf{u}^{(k)} \in \mathbb{C}^{M \times 1}$ is the Distributed Beamforming (DBF) vector verifying $\operatorname{Tr}\left\{\mathbf{u}^{(k)} \mathbf{u}^{(k) \mathrm{H}}\right\}=1$, whereas $\mathbf{p}^{(k)} \in \mathbb{R}^{M \times 1}$ is a vector that contains the power that each RAU participating in the JT-CoMP transmission allocates on carrier $k$.

Let $P_{\max , m}$ be the transmission power constraint of RAU $m$. Then, the corresponding centralized sum data rate optimization problem can be formally written as follows:

$$
\begin{array}{clr}
\underset{\mathbf{P a x i m i z e}}{\operatorname{m}, \mathbf{U}} & \sum_{k=1}^{K} \log _{2}\left(1+\bar{\gamma}^{(k)}\right) \\
\text { subject to } & \sum_{k=1}^{K} p_{m}^{(k)} \leq P_{\max , m} \quad m=1, \ldots, M \\
& p_{m}^{(k)} \geq 0 \quad k=1, \ldots, K ; m=1, \ldots, M \\
& \left\|\mathbf{u}^{(k)}\right\|=1 & k=1, \ldots, K
\end{array}
$$

with $\mathbf{P}=\left[\mathbf{p}^{(1)} \ldots \mathbf{p}^{(K)}\right]$ and $\mathbf{U}=\left[\mathbf{u}^{(1)} \ldots \mathbf{u}^{(K)}\right]$. Note that the objective function in (P1) is separable in the carrier domain, and DBF vector selection is uncoupled with respect to carrier index $k$. However, the sum power constraint is coupled in the carrier domain. Therefore, after selecting the optimal DBF vector per carrier, the optimal power allocation should be done in a cooperative way among all RAUs (i.e., optimal $\widehat{\mathbf{p}}_{m}$ depends on optimal $\widehat{\mathbf{p}}_{n}$ for all $n \neq m$ ). Note that optimal DBF vector is the eigenvector that corresponds to the maximum eigenvalue of the channel covariance matrix $\left(\mathbf{h}^{(k)} \mathbf{h}^{(k) \mathrm{H}}\right)$.

\section{Co-Phasing And Joint Power Allocation}

Our goal is to find the DBF vector per carrier (columns of matrix $\mathbf{U}$ ) and the power allocation profiles per RAU (rows of matrix $\mathbf{P}$ ) that maximize the objective function of (P1) when RAUs have only local CSI. For this purpose, we divide the sum data rate optimization problem into two parts:

1) Find the DBF vector $\widehat{\mathbf{u}}^{(k)}=\left[\widehat{u}_{1}^{(k)} \ldots \widehat{u}_{M}^{(k)}\right]^{\mathrm{T}}$ per carrier using $K$ QCP transmission modes in parallel.

2) Find the power allocation profile $\widehat{\mathbf{p}}_{m}=\left[\widehat{p}_{m}^{(1)} \ldots \widehat{p}_{m}^{(K)}\right]$ per RAU using a decentralized JPA algorithm.

The performance loss for using QCP instead of DBF with full CSI in transmission is expected to be minimal [9], [5].

\section{A. Quantized Co-Phasing Transmission Mode}

Quantized Co-Phasing adjusts the instantaneous phase of the signal transmitted from each RAU in discrete steps, such that the phase difference between the received copies is minimized. When all phase adjustments are done using RAU 1 as reference, the solution of this problem is obtained as follows [9]:

$$
\widehat{u}_{m}^{(k)}= \begin{cases}1 & \text { for } m=1, \\ e^{-j \widehat{\phi}_{m}^{(k)}} & \text { for } m=2, \ldots, M,\end{cases}
$$

where

$$
\widehat{\phi}_{m}^{(k)}=\arg \max _{\phi \in \mathcal{Q}}\left|h_{1}^{(k)}+h_{m}^{(k)} e^{-j \phi}\right| \quad m=2, \ldots, M
$$

is obtained using the phase quantization set

$$
\mathcal{Q}=\left\{\frac{(2 n-1) \pi}{2^{N_{p}}}: n=1, \ldots, 2^{N_{p}}\right\}
$$


that corresponds when $N_{p}$ phase bits are used per RAU. In practice this can be implemented using a decentralized algorithm, where each transmission point tests sequentially the different phase shifts in set $\mathcal{Q}$, and the MS informs the index that maximizes the received SNR on each carrier (see Fig. 1).

\section{B. Optimization of Joint Power Allocation}

After the DBF optimization part of problem (P1) is over, it is time that each RAU $m$ determines the optimal transmission power per carrier. Let $\mathbf{p}_{m}=\left[p_{m}^{(1)} \ldots p_{m}^{(K)}\right]$ be the initial power profile vector for RAU $m$. Then, the JPA optimization part of (P1) when $\widehat{\mathbf{U}}=\left[\widehat{\mathbf{u}}^{(1)} \ldots \widehat{\mathbf{u}}^{(K)}\right]$ is given can be written as

$$
\underset{\Delta \mathbf{P}}{\operatorname{maximize}} \sum_{k=1}^{K} \log _{2}\left(1+\frac{\left|\sum_{m=1}^{M} \sqrt{p_{m}^{(k)}+\Delta p_{m}^{(k)}} h_{m}^{(k)} \widehat{u}_{m}^{(k)}\right|^{2}}{P_{\mathrm{N}}}\right)
$$

subject to $\sum_{k=1}^{K} \Delta p_{m}^{(k)} \leq P_{\max , m}-\sum_{k=1}^{K} p_{m}^{(k)} \quad m=1, \ldots, M$

$$
\Delta p_{m}^{(k)} \geq-p_{m}^{(k)} \quad k=1, \ldots, K ; m=1, \ldots, M,
$$

where $\Delta \mathbf{p}_{m}=\left[\Delta p_{m}^{(1)} \ldots \Delta p_{m}^{(K)}\right]$ is the $m$-th row of matrix $\Delta \mathbf{P}$, which contains the differential power profile that RAU $m$ should apply on top of $\mathbf{p}_{m}$ to optimize the sum data rate.

Let $h_{m}^{(k)}=\left|h_{m}^{(k)}\right| e^{j \psi_{m}^{(k)}}$. Then, received SNR on carrier $k$ is

$$
\begin{aligned}
\gamma^{(k)} & =\frac{1}{P_{\mathrm{N}}}\left|\sum_{m=1}^{M} \sqrt{p_{m}^{(k)}+\Delta p_{m}^{(k)}}\right| h_{m}^{(k)}\left|e^{j\left(\psi_{m}^{(k)}-\widehat{\phi}_{m}^{(k)}\right)}\right|^{2} \\
& =\frac{1}{P_{\mathrm{N}}}\left[\sum_{m=1}^{M}\left(p_{m}^{(k)}+\Delta p_{m}^{(k)}\right)\left|h_{m}^{(k)}\right|^{2}\right. \\
& +2 \sum_{m_{1}=1}^{M-1} \sum_{m_{2}=m_{1}+1}^{M} \sqrt{p_{m_{1}}^{(k)}+\Delta p_{m_{1}}^{(k)}} \sqrt{p_{m_{2}}^{(k)}+\Delta p_{m_{2}}^{(k)}} \\
& \left.\times\left|h_{m_{1}}^{(k)}\right|\left|h_{m_{2}}^{(k)}\right| \cos \left(\theta_{m_{1}, m_{2}}^{(k)}\right)\right],
\end{aligned}
$$

where $\theta_{m_{1}, m_{2}}^{(k)}=\left(\psi_{m_{1}}^{(k)}-\widehat{\phi}_{m_{1}}^{(k)}\right)-\left(\psi_{m_{2}}^{(k)}-\widehat{\phi}_{m_{2}}^{(k)}\right)$ is a Random Variable (RV) that models the phase uncertainty that remains between the signals originated from RAU $m_{1}$ and $m_{2}$ after applying $N_{p}$-bit QCP on carrier $k$. It can be shown that the Probability Density Function (PDF) of RV $\theta_{m_{1}, m_{2}}^{(k)}$ is an even function, and that it is equivalent to the sum of two independent and identically distributed (i.i.d.) uniform RVs in the interval $\left[-\pi / 2^{N_{p}}, \pi / 2^{N_{p}}\right)$ when both $m_{1} \neq 1$ and $m_{2} \neq 1$. For $m_{1}=1$ or $m_{2}=1$, on the other hand, $\operatorname{RV~} \theta_{m_{1}, m_{2}}^{(k)}$ is uniformly distributed in the same interval [9].

In absence of a centralized unit that finds optimal $\Delta \mathbf{P}$ in a single iteration, we now derive a decentralized power allocation algorithm that maximizes the sum data rate distributively.

\section{DECENTRALIZED JOINT POWER ALLOCATION ALGORITHM}

This section solves (P2) using a decentralized JPA algorithm. When the transmission power profile vector of RAU $m$ does not change significantly between consecutive iterations, first-order Taylor series expansion

$$
\sqrt{p_{m}^{(k)}+\Delta p_{m}^{(k)}} \approx \sqrt{p_{m}^{(k)}}+\frac{1}{2} \frac{\Delta p_{m}^{(k)}}{\sqrt{p_{m}^{(k)}}} \quad \begin{aligned}
& k=1, \ldots, K \\
& m=1, \ldots, M
\end{aligned}
$$

can be used to obtain an accurate approximation. Then, after plugging (9) in (8) and applying the expectation operator,

$$
\bar{\gamma}^{(k)}=\mathbb{E}\left\{\gamma^{(k)}\right\} \approx \alpha^{(k)}+\sum_{m=1}^{M} \beta_{m}^{(k)} \Delta p_{m}^{(k)} \quad k=1, \ldots, K
$$

results, where

$$
\begin{aligned}
\alpha^{(k)} & =\frac{1}{P_{\mathrm{N}}}\left[\sum_{m=1}^{M} p_{m}^{(k)} \mathbb{E}\left\{\left|h_{m}^{(k)}\right|^{2}\right\}+2 \sum_{m_{1}=1}^{M-1} \sum_{m_{2}=m_{1}+1}^{M} \sqrt{p_{m_{1}}^{(k)}} \sqrt{p_{m_{2}}^{(k)}}\right. \\
& \left.\times \mathbb{E}\left\{\left|h_{m_{1}}^{(k)}\right|\right\} \mathbb{E}\left\{\left|h_{m_{2}}^{(k)}\right|\right\} \mathbb{E}\left\{\cos \left(\theta_{m_{1}, m_{2}}^{(k)}\right)\right\}\right] \quad k=1, \ldots, K(11)
\end{aligned}
$$

is a common factor for all RAUs on carrier $k$, while

$$
\begin{aligned}
\beta_{m}^{(k)} & =\frac{1}{P_{\mathrm{N}}}\left[\mathbb{E}\left\{\left|h_{m}^{(k)}\right|^{2}\right\}+\sum_{m_{2} \neq m} \sqrt{p_{m_{2}}^{(k)} / p_{m}^{(k)}} \mathbb{E}\left\{\left|h_{m}^{(k)}\right|\right\} \mathbb{E}\left\{\left|h_{m_{2}}^{(k)}\right|\right\}\right. \\
& \left.\times \mathbb{E}\left\{\cos \left(\theta_{\left(m, m_{2}\right)}^{(k)}\right)\right\}\right] \quad k=1, \ldots, K ; m=1, \ldots, M .(12)
\end{aligned}
$$

Note that in (11) and (12), $\mathbb{E}\left\{\left|h_{m}^{(k)}\right|^{2}\right\}$ combines the effect of spreading loss and molecular absorption on $\mathrm{THz}$ carrier $k$, whereas $\mathbb{E}\left\{\cos \left(\theta_{m_{1}, m_{2}}^{(k)}\right)\right\}$ estimates the effect of co-phasing uncertainty due to limited CSI. Based on [9], we have that

$\mathbb{E}\left\{\cos \left(\theta_{m_{1}, m_{2}}^{(k)}\right)\right\}= \begin{cases}\left(\frac{2^{N_{p}}}{\pi}\right) \sin \left(\frac{\pi}{2^{N_{p}}}\right) & \text { for } m_{1}, m_{2}=1 \\ \left(\frac{2^{2 N_{p}-1}}{\pi^{2}}\right)\left[1-\cos \left(\frac{\pi}{2^{N_{p}-1}}\right)\right] & \text { otherwise. }\end{cases}$

Based on the analysis of the Karush-Kuhn-Tucker (KKT) conditions of centralized problem (P2), it can be shown that its optimization is equivalent to solve simpler decentralized optimization problems (one per RAU) sequentially, i.e.,

$$
\begin{array}{cl}
\underset{\Delta \mathbf{p}_{m}}{\operatorname{maximize}} & \sum_{k=1}^{K} \log _{2}\left(\frac{1+\alpha^{(k)}}{\beta_{m}^{(k)}}+\Delta p_{m}^{(k)}\right) \quad m=1, \ldots, M \\
\text { subject to } & \sum_{k=1}^{K} \Delta p_{m}^{(k)} \leq P_{\max , m}-\sum_{k=1}^{K} p_{m}^{(k)} \\
& \Delta p_{m}^{(k)} \geq-p_{m}^{(k)} \quad k=1, \ldots, K .
\end{array}
$$

This is because the KKT conditions of both centralized problem (P2) and decentralized problems in (P3) are identical.

Solution of problem (P3) is known, and resembles the traditional waterfilling solution with slight modifications, i.e.,

$$
\Delta p_{m}^{(k)^{\star}}=\max \left\{-p_{m}^{(k)},\left[\frac{1}{\nu_{m}{ }^{\star}}-\frac{1+\alpha^{(k)}}{\beta_{m}^{(k)}}\right]\right\},
$$

where the waterfilling level $1 / \nu_{m}{ }^{*}$ is selected to verify

$$
\sum_{k=1}^{K} \max \left\{-p_{m}^{(k)},\left[\frac{1}{\nu_{m}^{\star}}-\frac{1+\alpha^{(k)}}{\beta_{m}^{(k)}}\right]\right\} \leq P_{\max , m}-\sum_{k=1}^{K} p_{m}^{(k)} \text {. }
$$

Convergence is analyzed after all RAUs update their power profile in the carrier domain. In practice, convergence is reached when an infinitesimal variation is observed in the elements of the power vector of all RAUs between consecutive iterations. Mathematically, this is equivalent to satisfy

$$
\max _{m=1, \ldots, M}\left\{\frac{\max _{k=1, \ldots, K}\left\{\left|p_{m}^{(k)}[i]-p_{m}^{(k)}[i-1]\right|\right\}}{P_{\max , m}}\right\} \leq \varepsilon_{\max }
$$

where $\varepsilon_{\max }$ is the maximum relative convergence tolerance. Convergence properties of this decentralized JPA algorithm 


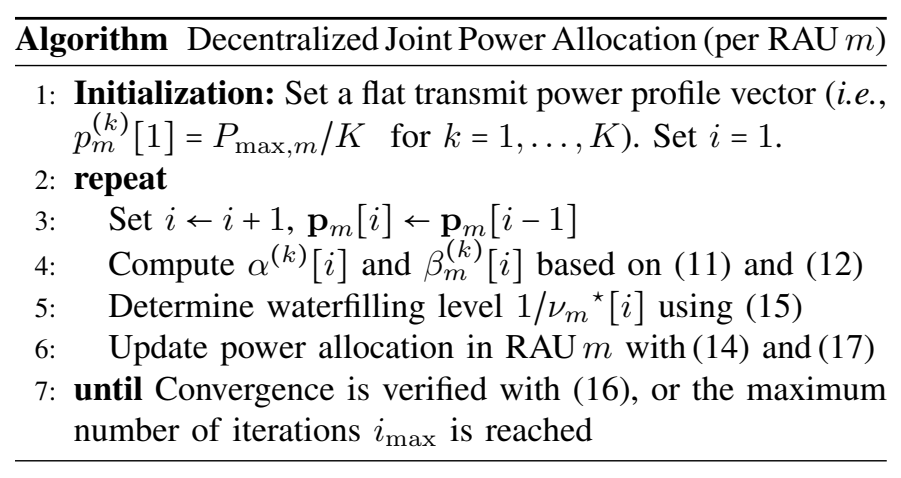

can be controlled updating the transmission power vectors with controlled step sizes using linear combination

$$
p_{m}^{(k)}[i]=p_{m}^{(k)}[i-1]+\delta_{m} \Delta p_{m}^{(k)^{\star}},
$$

where $\Delta p_{m}^{(k)^{\star}}$ is the solution of optimization problem (P3) on carrier $k, p_{m}^{(k)}[i-1]$ is the power allocated on carrier $k$ in the previous iteration (i.e., before the updating step is executed), and $\delta_{m}$ is the step size for RAU $m$ that guarantees a good tradeoff between convergence probability and convergence speed. A summary of this decentralized JPA algorithm, which runs asynchronously in each RAU $m$ participating in the JTCoMP communication, is presented on the top of this page.

\section{Simulation Results}

This section characterizes the sum data rate of JT-CoMP in presence of multiple frequency-selective $\mathrm{THz}$ carriers. We assume that $M$ RAUs apply $N_{p}$-bit QCP to provide coherent combining gain on $K$ equal-size carriers and, on top of that, different power allocation algorithms are used to distribute the limited transmission power. Three different power allocation algorithms are analyzed: Equal Power Allocation (EPA), Independent Waterfilling (IWF), and decentralized Joint Waterfilling (JWF). Note that IWF allocates transmission power among carriers like if RAU $m$ were the only active point.

The attenuation that a $\mathrm{THz}$ radio signal experiences during propagation can be divided into two components. On one hand, traditional free space spreading loss, which is given by

$L_{\text {prop }, m}[\mathrm{~dB}]=20 \log _{10}(4 \pi / \mathrm{c})+20 \log _{10}\left(f_{c}\right)+20 \log _{10}\left(d_{m}\right)$,

where $\mathrm{c}=3 \times 10^{8}[\mathrm{~m} / \mathrm{s}]$ is the speed of light, $f_{c}[\mathrm{~Hz}]$ is the carrier frequency, and $d_{m}[\mathrm{~m}]$ is the distance between the RAU $m$ and the MS. On the other hand, we have the molecular absorption loss $L_{\mathrm{abs}, m}[\mathrm{~dB}]=A_{f} d_{m}$, which depends on the concentration of water vapor and atmosphere gases, as well as their corresponding frequency of resonance. Molecular absorption is not easy to model, and has a frequency-selective behavior that grows notably with distance. In this paper, we use ITU-R recommendation [13] to estimate the attenuation of atmospheric gases and water vapor in terrestrial links. Therefore, if we assume that $K=9$ equal-spaced carriers exist between 300 and $500 \mathrm{GHz}$, then the molecular absorption losses in $[\mathrm{dB} / \mathrm{km}]$ are: $A_{300}=5, A_{325}=40, A_{350}=10$, $A_{375}=300, A_{400}=20, A_{425}=30, A_{450}=300, A_{475}=70$, and $A_{500}=60$. The carrier bandwidth is $W=1 \mathrm{GHz}$, the maximum transmission power per RAU is $P_{\max , m}=20 \mathrm{dBm}$, and $P_{N}$

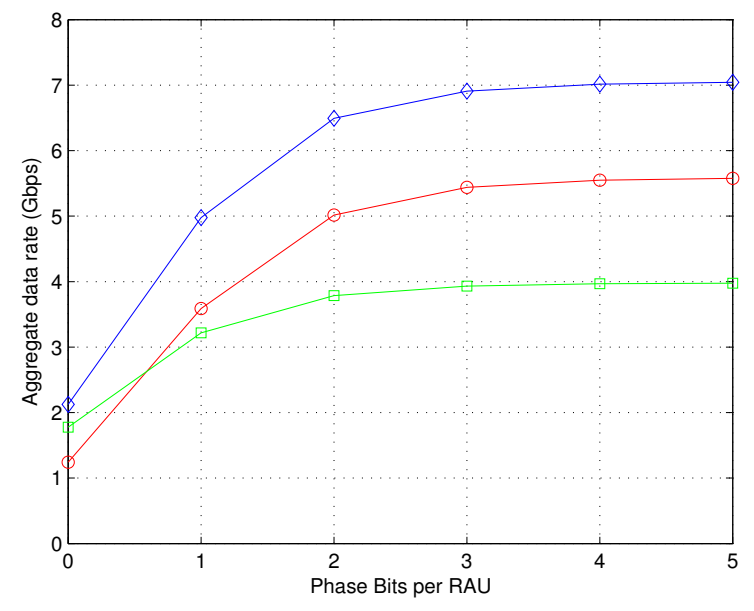

Fig. 2: Sum data rate as function of number of co-phasing bits per RAUs ( $K=9$ carriers, $W=1 \mathrm{GHz}, P_{\max , m}=20 \mathrm{dBm}, G_{\mathrm{tx}}=10 \mathrm{dBi}$, $M=6$ RAUs, $d=60 \mathrm{~m}$ ). Red line with circles: EPA. Green line with squares: IWF. Blue line with diamonds: Decentralized JWF.

contains only thermal noise at $290 \mathrm{~K}$ (ambient temperature). Moreover, antenna arrays in transmission and reception have a gain of 10 and $0 \mathrm{dBi}$, respectively. Note that these values are kept low on purpose, to highlight the benefits of JT-CoMP in presence of multiple wideband carriers. Step size and convergence criterion are $\delta_{m}=0.1$ and $\varepsilon_{\max }=0.01$, respectively.

Figure 2 shows the sum data rate of $N_{p}$-bit QCP when different power allocation algorithms are used. In this scenario, $M=6$ RAUs coordinate their transmission with a variable number of phase bits per RAU. As expected, decentralized JWF provides the best sum data rate performance. Since IWF concentrates the transmission power in the few carriers with lowest molecular absorption, the JT-CoMP system tends to work on a high-SNR regime with low (aggregate) communication bandwidth. The opposite situation takes place with EPA and, due to that, the sum data rate of EPA is (usually) higher than IWF. As expected, the achievable data rate increases as $N_{p}$ grows for all power allocation algorithms. In general terms, this gain saturates when $N_{p}=3$ phase bits are used. On the other hand, the absence of co-phasing information (i.e., $N_{p}=0$ ) affects notably the sum data rate since no coherent combining gain is exploited. To sum up, it could be said that $N_{p}=2$ gives the best tradeoff solution between performance gain and co-phasing feedback overhead required per RAU.

Figure 3 shows the sum data rate of JT-CoMP when different power allocation algorithms are combined with a variable number of RAUs ( $N_{p}=2$ in all cases). As expected, the sum data rate grows with the number of participating RAUs. Note that when $M$ is low, the total transmission power from active RAUs is limited; therefore, only few carriers with negligible molecular absorption are activated, making the sum data rate of IWF and JWF very similar. However, as the total transmission power grows with $M$, the activation of new carriers with higher molecular absorption starts to play a key role in the sum data rate maximization. This is the reason why EPA gives a better performance than IWF for large $M$. Moreover, though the data rate of IWF saturates as $M$ grows, the achievable data rate of EPA and JWF does not show any saturation for the number of RAUs under study. Note that 


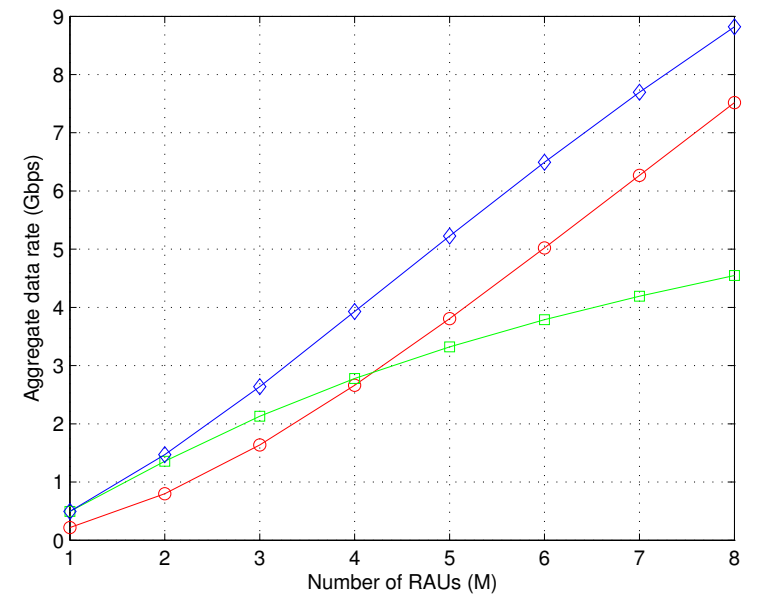

Fig. 3: Sum data rate as function of number of active RAUs $(K=9$ carriers, $W=1 \mathrm{GHz}, P_{\max , m}=20 \mathrm{dBm}, G_{\mathrm{tx}}=10 \mathrm{dBi}, d=60 \mathrm{~m}$, $N_{p}=2$ bits/RAU). Red line with circles: EPA. Green line with squares: IWF. Blue line with diamonds: Decentralized JWF.

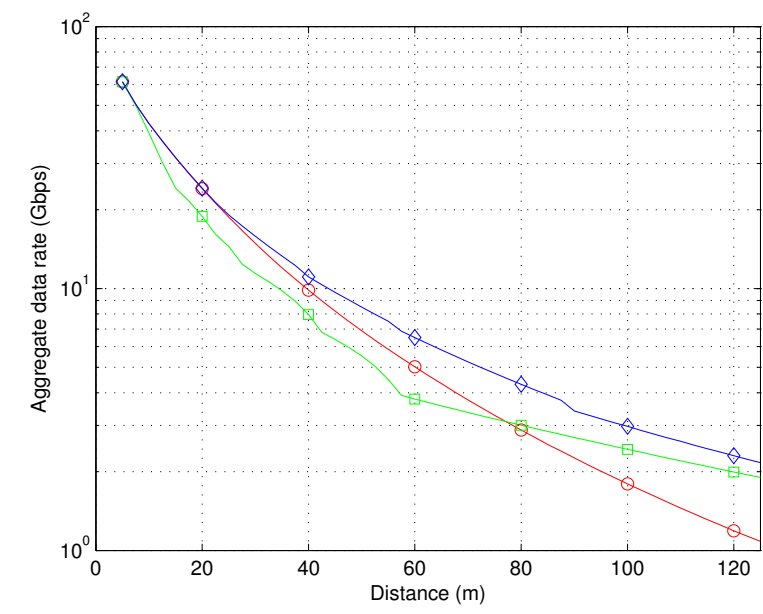

Fig. 4: Sum data rate as function of distance between RAUs and MS ( $K=9$ carriers, $W=1 \mathrm{GHz}, P_{\max , m}=20 \mathrm{dBm}, G_{\mathrm{tx}}=10 \mathrm{dBi}, M=6$ RAUs, and $N_{p}=2$ bits/RAU). Red line with circles: EPA. Green line with squares: IWF. Blue line with diamonds: Decentralized JWF.

JWF provides notable gains over EPA because its takes into account the effect of the frequency-selective THz channels and the power allocation profile that the other RAUs apply.

Finally, Fig. 4 characterizes the sum data rate of JT-CoMP for different distances between RAUs and MS ( $M=6$ and $N_{p}=2$ in all cases). Note that in the $\mathrm{THz}$ band, molecular absorption loss grows much more than spreading loss with distance. That is, if we measure both losses in $\mathrm{dBs}$, molecular absorption grows linearly with $d$, whereas spreading loss follows a $\log _{10}(d)$ relation. Due to that, the frequency-selective behavior of a $\mathrm{THz}$ channel vanishes at low $d$ (flat frequency response), and all power allocation algorithms tend to the same data rate performance. The opposite situation takes place at longer distances, justifying the use of power allocation based on waterfilling. More precisely, the sum data rate is maximized if the available transmission power is jointly allocated using the decentralized JWF algorithm proposed in Section IV. Note that when distance grows large, IWF and JWF tend to provide the same sum data rate performance since in both cases the carrier with lowest molecular absorption is the only active one.

\section{CONCLUSION}

A multi-carrier JT-CoMP scheme was proposed as enabler of extremely-high data rate communication in presence of a large number of transmission points. The effect of distance on the frequency-selective channel response was considered to affect the number of active $\mathrm{THz}$ carriers in the system. A decentralized multi-carrier JT-CoMP scheme that combines both JPA and $N_{p}$-bit QCP was formulated. Based on obtained simulation results, it was observed that the combination of JPA with QCP provides notable performance gains with respect to non-cooperative power allocation strategies (EPA and IWF). As expected, the accuracy of reported channel phase information, the level of distance-dependent frequency selectivity in the $\mathrm{THz}$ carriers, and the number of RAUs participating in the coordinated transmission affected the sum data rate that multicarrier JT-CoMP was able to provide. These results support the use of the $\mathrm{THz}$ band for novel applications, such as the provision of high-speed mid-range wireless backhaul links to enable future ultra-dense deployments of small cell networks.

\section{ACKNOWLEDGMENT}

This work has received funding from the Spanish Government under Projects TEC2014-59255-C3-1-R and TEC2014-59255-C3-3-R, the Catalan Government under Grant 2014SGR1567, and the Spanish MECD FPU fellowship program granted to the co-author B. Genovés Guzmán.

\section{REFERENCES}

[1] I. F. Akyildiz, J. M. Jornet, and C. Han, "Terahertz band: Next frontier for wireless communications," Physical Communication, vol. 12, pp. 16-32, Sept. 2014.

[2] C. Han, A. O. Bicen, and I. F. Akyildiz, "Multi-wideband waveform design for distance-adaptive wireless communications in the terahertz band," IEEE Trans. Signal Process., vol. 64, no. 4, pp. 910-922, Feb. 2016.

[3] A. Moldovan, M. A. Ruder, I. F. Akyildiz, and W. H. Gerstacker, "LOS and NLOS channel modeling for terahertz wireless communication with scattered rays," in Proc. IEEE Global Commun. Conf. Workshops, Dec. 2014, pp. 388-392.

[4] R. Irmer et al., "Coordinated multipoint: Concepts, performance, and field trial results," IEEE Commun. Mag., vol. 49, no. 2, pp. 102-111, Feb. 2011.

[5] B. Haile, A. Dowhuszko, J. Hämäläinen, R. Wichman, and Z. Ding, "On performance loss of some CoMP techniques under channel power imbalance and limited feedback," IEEE Trans. Wireless Commun., vol. 14 , no. 8, pp. 4469-4481, Aug. 2015.

[6] B. Luo, Q. Cui, H. Wang, and X. Tao, "Optimal joint water-filling for OFDM systems with multiple cooperative power sources," in Proc. IEEE Global Commun. Conf., Dec. 2010, pp. 1-5.

[7] Q. Cui, B. Luo, X. Huang, A. Dowhuszko, and J. Jiang, "Closed-form solution for minimizing power consumption in coordinated transmissions," Eurasip J. Wireless Commun. Netw., vol. 2012, pp. 1-14, Mar. 2012.

[8] D. Love et al., "An overview of limited feedback in wireless communication systems," IEEE J. Sel. Areas Comm., vol. 26, no. 8, pp. 1341-1365, Oct. 2008

[9] J. Hämäläinen, R. Wichman, A. Dowhuszko, and G. Corral-Briones, "Capacity of generalized UTRA FDD closed-loop transmit diversity modes," Wireless Personal Communications, vol. 54, pp. 467-484, Aug. 2010.

[10] A. Dowhuszko and J. Hämäläinen, "Performance of transmit beamforming codebooks with separate amplitude and phase quantization," IEEE Signal Process. Letters, vol. 22, no. 15, pp. 813-817, Jul. 2015.

[11] Ya-Hui Hu and Geng-Sheng Kuo, "Space-time-frequency domain water-filling in MIMO-OFDM fading system," in Proc. IEEE Veh. Tech. Conf. Spring, Apr. 2007, pp. 2475-2480.

[12] D. Wang et al., "Spectral efficiency of distributed MIMO systems," IEEE J. Sel. Areas Commun., vol. 31, no. 10, pp. 2112-2127, Oct. 2013.

[13] ITU-R, "Attenuation by atmospheric gases," Recommendation ITU-R P.676-10, pp. 1-22, Sept. 2013. 